\title{
Non-destructive dose verification of two drugs within 3D printed polyprintlets
}

Sarah J Trenfield ${ }^{1}$, Hui Xian Tan ${ }^{1}$, Alvaro Goyanes², David Wilsdon ${ }^{3}$, Martin Rowland ${ }^{4}$, Simon Gaisford ${ }^{1,2}$ and Abdul W Basit ${ }^{1,2 *}$

${ }^{1}$ UCL School of Pharmacy, University College London, 29-39 Brunswick Square, London WC1N 1AX, UK ${ }^{2}$ FabRx Ltd., 3 Romney Road, Ashford, Kent, TN24 ORW, UK ${ }^{3}$ Pfizer Ltd., Analytical Research and Development, Discovery Park, Ramsgate Road, Sandwich, CT13 9ND, UK

${ }^{4}$ Pfizer Ltd., Drug Product Design, Discovery Park, Ramsgate Road, Sandwich, CT13 9ND, UK

*Correspondence: a.basit@ucl.ac.uk (Abdul W. Basit)

Key words: 3D printing; Selective laser sintering; Process analytical technology (PAT); Oral drug delivery systems; Near infrared spectroscopy; Digital healthcare; Personalized medicines 


\begin{abstract}
Three-dimensional printing (3DP) is a revolutionary technology in pharmaceuticals, enabling the personalisation of flexible-dose drug products and 3D printed polypills (polyprintlets). A major barrier to entry of this technology is the lack of non-destructive quality control methods capable of verifying the dosage of multiple drugs in polyprintlets at the point of dispensing. In the present study, 3D printed films and cylindrical polyprintlets were loaded with flexible, therapeutic dosages of two distinct drugs (amlodipine and lisinopril) across concentration ranges of $1-5 \% w / w$ and $2-10 \% w / w$, respectively. The polyprintlets were non-destructively analysed for dose content using a portable near infrared (NIR) spectrometer and validated calibration models were developed using partial least squares (PLS) regression, which showed excellent linearity $\left(R^{2}\right.$ Pred $\left.=0.997,0.991\right)$, accuracy $(\mathrm{RMSEP}=0.24 \%, 0.24 \%)$ and specificity $(\mathrm{LV} 1=82.77 \%, 79.55 \%)$ for amlodipine and lisinopril, respectively. X-ray powder diffraction (XRPD) and thermogravimetric analysis (TGA) showed that sintering partially transformed the phase of both drugs from the crystalline to amorphous forms. For the first time, we report a non-destructive, RTR quality control of two separate active ingredients in a single 3D printed drug product using NIR spectroscopy, overcoming a major barrier to the integration of $3 \mathrm{D}$ printing into clinical practice.
\end{abstract}




\section{1. Introduction}

2 Hypertension is a silent killer, responsible for over 7.5 million deaths / year 3 (12.8\% of all deaths) worldwide (WHO, 2019). Polypharmacy, which signifies

4 the concurrent use of multiple medications by one individual, is the current gold standard for treating hypertension (Durden et al., 2013) (NICE, 2011). Due to polypharmacy, medication adherence is a major challenge in the management of hypertension, with over $65 \%$ of patients failing to adhere to their prescribed regimens, and $50 \%$ ceasing their medication regime within one year of prescription (Ruilope, 2011; Tibebu et al., 2017) (Abegaz et al., 2017; Corrêa et al., 2016).

Critically, a long-term non-adherence to treatment has been associated with an increased risk of cardiovascular events, including strokes, hospitalisations and death (Abegaz et al., 2017; Herttua et al., 2013; Lee et al., 2017; Ong et al., 2007). To resolves the issues arising from polypharmacy, the use of fixed drug combinations (also known as 'polypills') have been explored. However, although polypills have been shown to improve adherence, the dosage of each drug is fixed making changes in dosage regimens inconvenient (Roy et al., 2017). In order to overcome this, there is a need for a novel platform that enables flexible dosing for polypills and three-dimensional printing (3DP) has the potential to do so (Alomari et al., 2018; Trenfield et al., 2019a).

3DP has gained momentum in many industries such as the aeronautics, robotics, electronics, manufacturing and food industries and, more recently, within medicine and pharmaceuticals (Barnatt, 2013). Within the pharmaceutical field, it is no longer a new idea to transition away from the standard mass production of medicines of fixed strength towards creating personalised dosage forms and dose combinations (Awad et al., 2018a; Awad et al., 2018b; Goyanes et al., 2017). By creating medicines in a layer-by-layer manner, this technology can produce printlets (3D printed tablets) that are customised to a patient's disease state, individual factors and therapeutic needs (Florence and Lee, 2011; Goyanes et al., 2019b; Hamburg and Collins, 2010; Oblom et al., 2019; Trenfield et al., 2018a). Due to the ability for precise 
material deposition, several studies have demonstrated the potential for 3DP to create polypills (polyprintlets) containing more than one active pharmaceutical ingredient (API) (Genina et al., 2017; Gioumouxouzis et al., 2018; Khaled et al., 2015a, b; Robles-Martinez et al., 2019; Sadia et al., 2018b).

Whilst the evidence-based for 3DP of polyprintlets is increasing, the integration of this technology into clinical practice has not yet been achieved (Alhnan et al., 2016; Basit and Gaisford, 2018; Edinger et al., 2018). A major barrier preventing 3DP uptake into pharmaceuticals is the absence of an at-line, nondestructive quality control $(\mathrm{QC})$ techniques to enable the real-time release of 3D printed medicines (Di Prima et al., 2016; Trenfield et al., 2018b; Trenfield et al., 2019b). Analytical methods such as dose quantification using chromatographic methods, as well as dissolution and disintegration testing, are commonly used for QC of pharmaceuticals. However, these characterisation methods are inherently destructive, which would be inconvenient for individually fabricated printlets at the point-of-care (Awad et al., 2018a).

An alternative approach could involve the integration of real-time release (RTR) testing, which is the ability to evaluate and ensure the quality of in-process and/or final product based on process data (EMA, 2012). Vibrational spectroscopic tools, such as near infrared (NIR) spectroscopy combined with chemometrics, have previously been used as alternative QC tools within pharmaceutical processes (Edinger et al., 2019; Trenfield et al., 2018b; Vakili et al., 2017). NIR spectroscopy has been widely used for at-line analysis because it has the capability to analyse and quantify drugs in a rapid, nondestructive and user-friendly manner. Furthermore, it can be conveniently integrated at the point of dispensing in the clinic due to its portability. The potential for NIR spectroscopy as a non-destructive QC method was demonstrated by our group previously whereby a point-and-shoot approach was used to measure the drug content of paracetamol in printlets (Trenfield et al., 2018b). To date, previous research had predominantly focused on quantifying single active ingredients within individually fabricated dosage forms using NIR 

7 8

spectroscopy (Trenfield et al., 2018b). However, one of the major benefits of 3DP is the ability to produce polyprintlets containing multiple APIs in the same dosage form (Pereira et al., 2018; Xu et al., 2020). As such, for the first time, we demonstrate the non-destructive QC of two distinct APIs (lisinopril and amlodipine) at therapeutically-relevant dosages within 3D printed polyprintlets using a portable, reflectance NIR spectrometer. The applicability of the model to polyprintlets of different geometries (cylindrical and oral films) was evaluated, and dosage forms were characterised using $\mathrm{x}$-ray powder diffraction (XRPD) and thermogravimetric analysis (TGA) to elucidate drug distribution and solidstate characteristics.

\section{Materials and Methods}

Amlodipine (LKT Laboratories, Inc., US) (MW $408.879 \mathrm{~g} / \mathrm{mol}$, solubility at $25^{\circ} \mathrm{C}$ $75.3 \mathrm{mg} / \mathrm{L}$ ) (Pubchem, 2003) and lisinopril dihydrate (Acros Organics, UK) (MW $441.525 \mathrm{~g} / \mathrm{mol}$, solubility at $25^{\circ} \mathrm{C} 216 \mathrm{mg} / \mathrm{L}$ ) (DrugBank; Pubchem, 2005). Polyethylene oxide (PEO) 100,000 (Sigma-Aldrich, UK) which has a molecular weight of $100,000 \mathrm{~g} / \mathrm{mol}$ and density of $1.13 \mathrm{~g} / \mathrm{mL}$ (Pubchem, 2004) was used as the thermoplastic polymer in the sintering process. Candurin ${ }^{\circledR}$ Gold Sheen was purchased from Merck KGaA, Germany.

\subsection{D printed design}

Templates of the printlets were designed by using 123D Design Software (Autodesk Inc, UK), a computer-aided design (CAD) software to create 3D representations of the object. Oral square films $(10 \mathrm{~mm} \times 10 \mathrm{~mm} \times 0.5 \mathrm{~mm})$ and standard cylindrical printlets (10 mm diameter $\times 3.6 \mathrm{~mm}$ height) were designed.

3D models were exported as a stereolithographic (.stl) file into 3D printer Sintratec Central software Version 1.1.13.

\subsection{Printing process}


100 PEO 100,000 was sieved by using a $180 \mu \mathrm{m}$ orifice-size sieve to reduce its

101 particle size for printing. Twenty-five formulations were prepared for building 102 the calibration model for NIR spectroscopy across five different dosage 103 concentration ranges $(n=5$, Table 1). The lowest and highest concentrations 104 of both drugs were selected to enable the provision of therapeutically-relevant 105 dosages, i.e. assuming a 200mg tablet is produced, amlodipine $1-5 \% \mathrm{w} / \mathrm{w}$ 106 covers a $2 \mathrm{mg}-10 \mathrm{mg}$ dose range and lisinopril $2-10 \% \mathrm{w} / \mathrm{w}$ covers a $4 \mathrm{mg}-20 \mathrm{mg}$ 107 dose range. For each formulation, $15 \mathrm{~g}$ of a mixture of drugs and excipients 108 were blended using a pestle and mortar. $3 \% \mathrm{w} / \mathrm{w}$ of Candurin Gold Sheen was 109 added to all formulations to enhance absorption of the laser to allow printability 110 (Fina et al., 2017). The powder mixtures were transferred to a selective laser 111 sinter (SLS) printer (Sintratec Kit, AG, Brugg, Switzerland) for printing. The 112 chamber temperature (which indicates the temperature inside the printer body) 113 was maintained at $30{ }^{\circ} \mathrm{C}$ and the surface temperature (which refers to the 114 temperature of the powder bed surface in the build platform) was maintained at $11540{ }^{\circ} \mathrm{C}$. The laser scanning speed was set at $200 \mathrm{~mm} / \mathrm{s}$. The printing process 116 started with the activation of a $2.3 \mathrm{~W}$ blue diode laser (445 $\mathrm{nm}$ ) to sinter the 117 powder within the build platform in a certain pattern based on the stl file. 118 Powder in the reservoir platform $(150 \mathrm{~mm} \times 150 \mathrm{~mm} \times 30 \mathrm{~mm})$ of the printer 119 was moved by a sledge to a building platform (150 mm x $150 \mathrm{~mm} \times 30 \mathrm{~mm}$ ) 120 creating a flat and homogeneously distributed layer of powder. Then, the laser 121 would sinter on the powder particles together. This process was repeated layer122 by-layer until the object was completed. The dosage form was then removed 123 from the powder bed and excess powder was brushed off. Five oral films were 124 printed at the same time for each formulation. Three formulations (A2L4, A3L6 125 and A4L8) were chosen to be printed into cylindrical tablets for inclusion into 126 the building the calibration model $(n=3)$. 


\begin{tabular}{ccccc}
\hline Formulation code & $\begin{array}{c}\text { Drug concentration }(\% w / w) \\
\text { Amlodipine }\end{array}$ & $\begin{array}{c}\text { PEO } \\
(\% w / w)\end{array}$ & $\begin{array}{c}\text { Candurin } \\
\text { Gold Sheen } \\
(\% w / w)\end{array}$ \\
\hline A1L2 & 1 & 2 & 94 & 3 \\
A2L4 & 2 & 4 & 91 & 3 \\
A3L6 & 3 & 6 & 88 & 3 \\
A4L8 & 4 & 8 & 85 & 3 \\
A5L10 & 5 & 10 & 82 & 3 \\
\hline
\end{tabular}

134

135

136

137

138

139

140

\subsection{Near infrared spectroscopy (NIR) data acquisition}

A portable benchtop Labspec 5000 NIR spectrometer (Analytical Spectral Devices, USA), equipped with three separate holographic diffraction gratings and three separate detectors; a 512-element silicon photo-diode array for wavelengths between 350-1000 nm, and two TE-cooled InGaAs for wavelengths between $1000-1800 \mathrm{~nm}$ and $1800-2500 \mathrm{~nm}$ was used to measure the NIR reflectance. An immobilised lab grade $1 \mathrm{~m}$ fibre optic cable (fibre core size $200 \mu \mathrm{m}$ ), which interfaced with the NIR equipment (BIF200- Vis-NIR, Ocean Optics Inc., FL, USA) was used to collect the spectra. A Spectralon 99\% reflective standard (Labsphere, North Sutton, UK) was used for instrument calibration prior to spectra acquisition. UV-visible-NIR spectra were collected across the 350-2500 nm wavelength region (2150 data points) totalling 64 scans, which were averaged. Each printlet was analysed at six different points to avoid potential sampling errors and to reduce the variability caused by different surface effects. All printlets were scanned three times on each side with the same format. The final spectrum (used to calculate amlodipine and lisinopril content) was the average of the spectra recorded at the six positions ( 6 averaged spectra/tablet). The data was processed by using Microsoft Excel and MATLAB software version R2017a (The MathWorks, CA, USA).

\subsection{Model development}

All five oral film concentrations $(n=5)$ (amlodipine 1-5\% w/w; lisinopril 2-10\% $w / w$; Table 1) were selected for calibration model development. Two oral films from A2L4, A3L6 and A4L8 was used for internal validation. Multivariate data analysis was performed using MATLAB software version R2017a (The 
160 MathWorks, CA, USA) with the PLS Toolbox version 8.6 (Eigenvector, CA, USA)

161 for data pre-processing and modelling. Partial least squares (PLS) regression

162 was performed on the datasets to build calibration models. The models were

163 internally cross-validated using Venetian blinds. Validation of the NIR

164 calibration model was performed according to guidance from the International

165 Conference on Harmonization (ICH) guidance Q2(R1) (ICH, 1994b)), European

166 Medicines Agency (EMA) (EMA, 2014b) and the Food and Drug Administration

167 (FDA) (FDA, 2015a), by assessing model specificity, linearity (expressed as

168 correlation coefficient, $\mathrm{R}^{2}$ ) and accuracy (expressed as the root mean square

169 error of prediction; RMSEP). The calibration model developed covered a total

170 of 25 samples of oral films (with 19 samples being selected for calibration and

1716 samples for internal validation) over an amlodipine concentration range of 1 -

$1725 \% w / w$ and lisinopril concentration range of $2-10 \% w / w$.

173

\section{2.5. Determination of drug content}

175 Three individual oral film printlets of each formulation were placed in separate 176 volumetric flasks with deionised water and methanol (50:50). Samples of the 177 solution were then filtered using a $0.45 \mu \mathrm{m}$ membrane filter (Millipore Ltd., 178 Ireland) into the HPLC vials. HPLC analysis was performed using UV-HPLC 179 equipped with an Eclipse Plus C18 column (150 x $4.6 \mathrm{~mm}, 5 \mu \mathrm{m}$ particle size) 180 (Agilent, UK) at a temperature of $40^{\circ} \mathrm{C}$. Analyses were carried at a detection wavelength of $215 \mathrm{~nm}$, a flow rate of $1 \mathrm{~mL} / \mathrm{min}$, an injection volume of $100 \mu \mathrm{L}$ and a run time of 18 mins. The mobile phase consisted of a gradient of solvent

183 A (HPLC water adjusted to $\mathrm{pH} 3$ with phosphoric acid) and solvent $\mathrm{B}$ 184 (acetonitrile). The method entailed the following: 1) solvent $A$ and solvent $B$ 185 were set at 83:17 at the start time; 2) then adjusted to 80:20 at the $6^{\text {th }}$ minute; 186 3) then adjusted to $10: 90$ at the $15^{\text {th }}$ minute 4 ) adjusted to $83: 17$ at the $18^{\text {th }}$ 187 minute. Elution times for amlodipine and lisinopril were 1.9 mins and $11.1 \mathrm{mins}$, 188 respectively.

\subsection{X-ray powder diffraction}

192 A Rigaku MiniFlex 600 (Rigaku, USA) with a Cu Ka X-ray source $(\lambda=1.5418$

$193 \AA$ ) and accompanying software Miniflex Guidance version 1.2.01 were used to 
194

195

196

197

198

199

200

201

202

203

204

205

206

207

208

209

210

211

212

213

214

215

216

217

218

219

220

221

222

223

224

225

record x-ray powder diffraction (XRPD) patterns of printlets (ground to fine powder), formulation blends and pure amlodipine, lisinopril, PEO 100,000 and Candurin Gold. The intensity and voltage applied were $15 \mathrm{~mA}$ and $40 \mathrm{kV}$ respectively. The angular range of data acquisition was $3-40^{\circ} 2 \theta$, with a step size of $0.02^{\circ}$ at a speed of $2^{\circ} \min ^{-1}$.

\subsection{Thermal analysis}

Thermogravimetric analysis (TGA) was also used for characterisation. All the samples were heated at $10^{\circ} \mathrm{C}$ min until $400{ }^{\circ} \mathrm{C}$ in open aluminium pans using Discovery TGA (TA instruments, Waters, LLC, USA). The purge gas used was nitrogen gas with a flow rate of $25 \mathrm{~mL} / \mathrm{min}$. Data were collected and analysed by using TA Instruments Trios software and percentage mass loss and onset temperature were calculated. The results from thermal analysis were plotted using OriginPro Software (OriginPro 2017 (64 bit) SR2 b9.4.2.380).

\subsection{Characterisation of the printlets}

\subsubsection{Determination of printlet weight variability}

All cylindrical printlets were weighed by using a weighing balance (Sartorius AG CPA225D, Germany). Printlets were measured in triplicate, and the mean and standard deviation for each printlet was calculated.

\subsubsection{Determination of printlet strength}

A traditional tablet hardness tester TBH 200 (Erweka GmbH, Heusenstamm, Germany) was used to determine the crushing strength of three cylindrical printlets of each drug combination. The mean and standard deviation for each printlet was calculated.

\subsubsection{Determination of printlet friability}

Three tablets of each concentration were weighed and placed into the drum of a Friability Tester Erweka type TAR 10 (Erweka $\mathrm{GmbH}$, Heusenstamm, 
226 Germany). The drum was rotated at $25 \mathrm{rpm}$ for 100 rounds and the samples 227 were reweighed. The friability of these samples was analysed in terms of weight 228 loss and it was expressed as percentage of original sample weight.

\section{Results and Discussion}

231 For the first time, it was possible to use a low temperature SLS printing process

232 to manufacture 3D printed oral square films and cylindrical printlets containing 233 two drugs (amlodipine and lisinopril) at therapeutically relevant concentrations

234 (up to $5 \% w / w$ and $10 \% w / w$, respectively; Figure 1). Previously studies have 235 proven the feasibility of using SLS 3DP technology in the pharmaceutical field 236 by successfully manufacturing immediate and modified release tablets (Barakh 237 Ali et al., 2019; Fina et al., 2017; Fina et al., 2018a), as well as fast 238 disintegrating oro-dispersible tablets (Fina et al., 2018b). Awad et al. has also 239 shown the capability of SLS 3D printing to produce pellets (miniprintlets) 240 containing more than one drug (Awad et al., 2019). However, the majority of 241 these studies required the use of elevated temperatures $\left(80-135^{\circ} \mathrm{C}\right)$ to enable 242 effective sintering. Favourably, due to low $\mathrm{T}_{\mathrm{g}}$ of the polymer (PEO 100,000, $\mathrm{T}_{\mathrm{g}}$ 243 of $-67^{\circ} \mathrm{C}$ ), it was possible to manufacture the dosage forms at a low 244 temperature $\left(40^{\circ} \mathrm{C}\right)$, which could be highly beneficial for thermally-labile drugs 245 that are unsuitable for higher temperature 3D printing processes (Goyanes et al., 2015a).
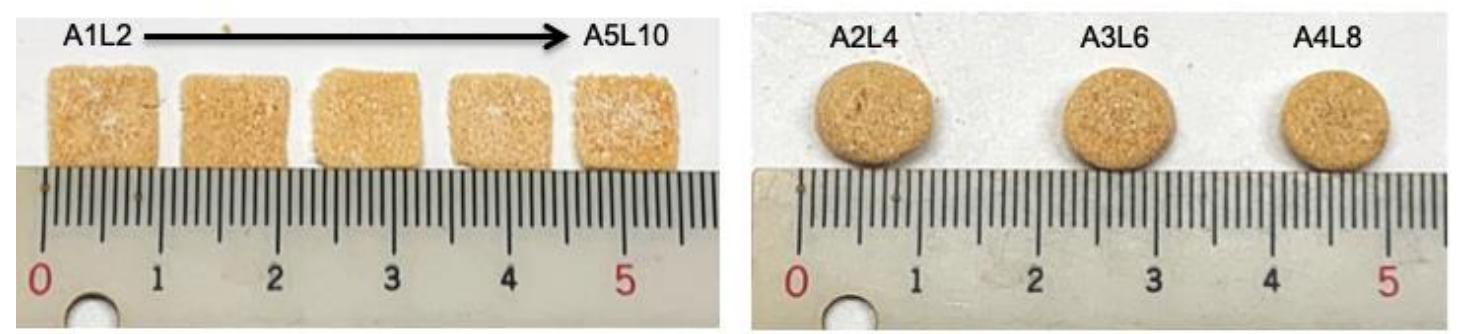

Figure 1. Printlets of two different geometries; square oral film (left) and cylindrical shape tablet (right). Drug content increases from left to right. The scale is in $\mathrm{cm}$.

\subsection{Polyprintlet characterisation}

254 Initially, TGA was performed to evaluate whether the drugs would be stable at 255 the temperatures required during the sintering process (Figure 2). The results 
256 showed that lisinopril decomposition occurred gradually in three steps. A weight 257 loss of $\sim 8 \%$ was observed up to $100^{\circ} \mathrm{C}$, attributed to the loss of water due to 258 lisinopril being in the dihydrate form. A constant weight was maintained 259 between $100-175^{\circ} \mathrm{C}$ indicated that no alteration occurred in the dehydrated 260 lisinopril crystal during this stage. Beyond $175^{\circ} \mathrm{C}$, the lisinopril crystal melted $261\left(\mathrm{~T}_{\mathrm{m}}=178-179^{\circ} \mathrm{C}\right)$ and degraded, which is similar to findings reported in the 262 literature (Hinojosa-Torres et al., 2008). TGA data of the other components 263 (amlodipine and PEO 100,000) and the formulation blends predicted that all the 264 components would remain stable and no degradation of the drugs and 265 excipients was likely to occur at the printing temperatures $\left(40^{\circ} \mathrm{C}\right)$. HPLC 266 analysis was also used to confirm stability of amlodipine and lisinopril post267 printing, with the HPLC trace showing only evidence of the main APIs peaks 268 after sintering.

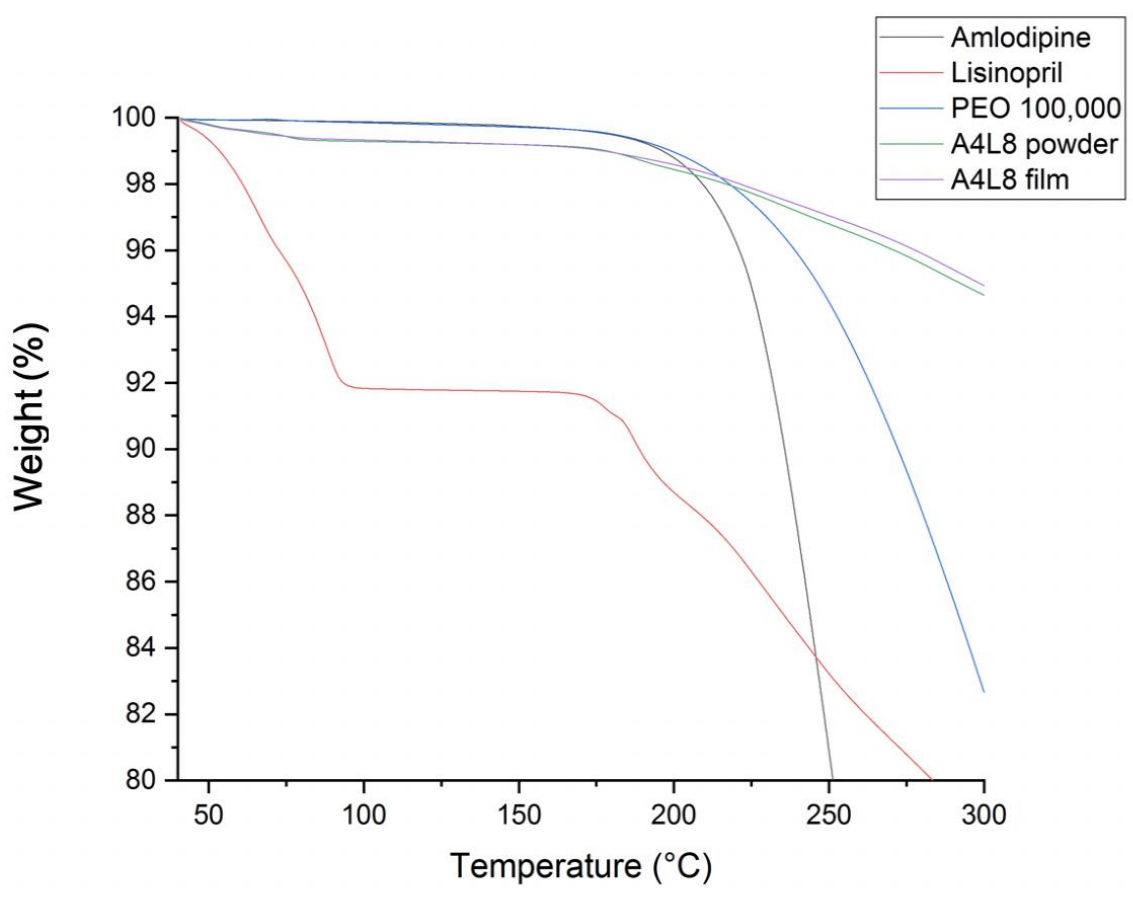

Figure 2. Thermogravimetric analysis of amlodipine, lisinopril, PEO 100,00, A4L8 formulation blend and A4L8 film.

272

273 XRPD analysis of the drug, polymers, formulation blends and printlets were 274 performed to determine the physical state of the drugs and the degree of 275 incorporation within the polymers (Figure 3). Characteristic patterns from the 276 XRPD focused between 3 to $16^{\circ} 2 \theta$ showed that the lisinopril drug peak present 
at about $7.5^{\circ} 2 \theta$ was also present in the powder blend. However, in the films, the peak became broader and showed a significant reduction in peak height. This indicates that lisinopril had been converted partially into the amorphous phase. Characteristic amlodipine peaks were present at $10^{\circ}$ and $12^{\circ} 2 \theta$ in the powder formulation but not the printed film indicating that either there was a complete conversion to the amorphous state or, alternatively, the remaining crystalline content was below the sensitivity of the XRPD method. Consistent drug and polymer peak shifts of $\sim+1^{\circ} 2 \theta$ was apparent in the printed formulation, which was attributed to the stress-strain influence or the change in height presentation of a printed disc versus the raw powder. Such peak shifts have been observed in previous studies (Robles-Martinez et al., 2019).

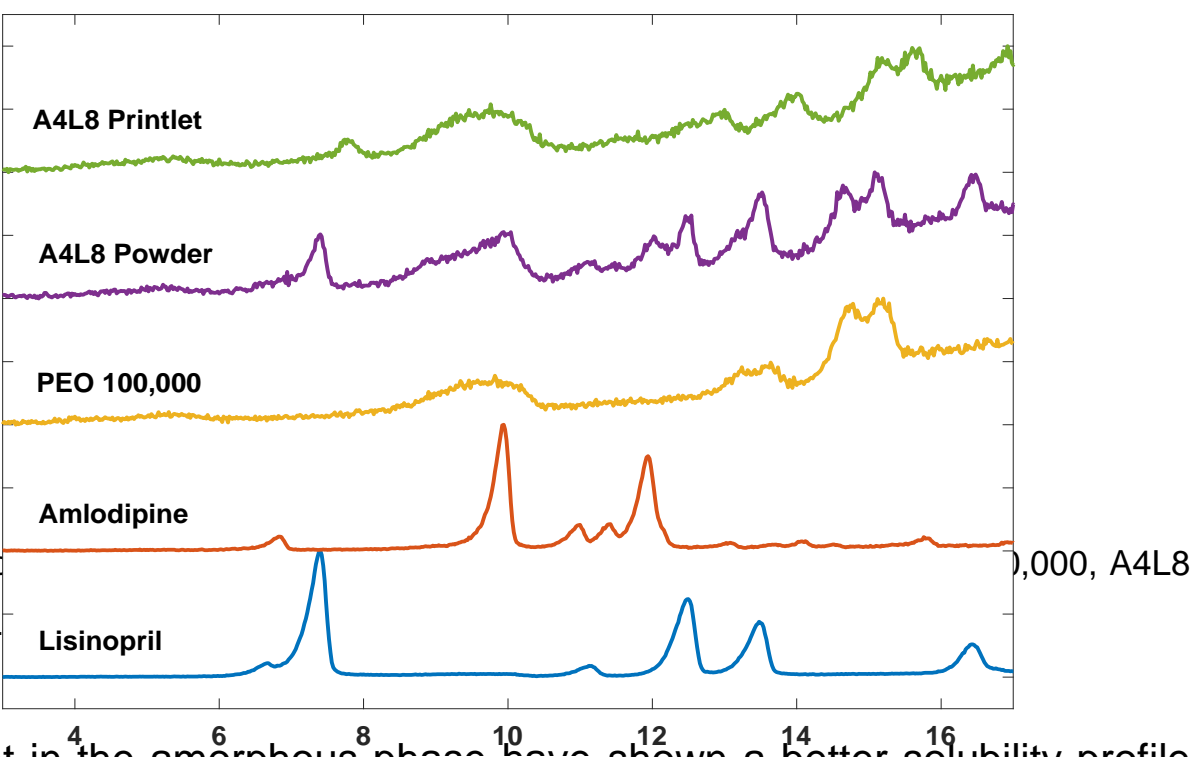

Drugs present in the amórphous ${ }^{8} \mathrm{pr}_{2 \theta}\left(^{\circ}\right)$ shown a better solubility profile compared with crystalline drugs (Balvu aı iu r vangia, 2011). Therefore, there are a number of advantages of delivering drugs in an amorphous form especially for drugs under BCS Class II or IV that has low solubility to enhance its dissolution and bioavailability (Capretto et al., 2017; Martinez et al., 2014). Several papers have shown the feasibility of using 3D printing technologies to formulate drugs in amorphous or semi-amorphous states to achieve enhanced drug release profiles (Goyanes et al., 2019a; Kollamaram et al., 2018; Sadia et al., 2016). During the SLS process used here, the application of the laser may 
311 lead to complete drug melting to enable formation of the non-crystalline

312 matrices (Trenfield et al., 2018b). However, this process will vary based on the

313 drugs, excipients and printing parameters used, such as laser scanning speed

314 and chamber temperature. It is worth mentioning however, that despite the

315 benefits of formulating drugs in the amorphous phase, there could also be a

316 risk of conversion back to its crystalline state and hence in the future

317 accelerated stability studies are required to determine shelf life of the 3D printed

318 drug products.

320 Printlet hardness was evaluated to determine the ease of handling of the 321 developed formulations. For all the cylindrical printlets, the hardness exceeded 322 the maximum value that the equipment could measure because the printlets 323 did not break but they were physically deformed (Table 2). Friability of all the

324 formulations of cylindrical printlets were less than $1 \%$, complying with the British

325 Pharmacopoeia (BP) requirements for uncoated tablets, making them suitable 326 for handling and packing (BP, 2018). Favourably, percentage recoveries of both 327 amlodipine and lisinopril were determined using HPLC, and were all found to 328 be between the $85-115 \%$ limits that have been set by BP for content uniformity 329 testing (Table 2). All dosage forms were found to pass weight variation tests 330 according to the $\mathrm{BP}(<7.5 \%$ variation).

331

332 Table 2. Physical properties and recovery of the cylindrical printlets

333

334

335 3.2. Quantitative analysis using NIR spectroscopy

\begin{tabular}{cccccc}
\hline Formulation & $\begin{array}{c}\text { Weight }(\mathrm{mg}) \pm \\
\mathrm{SD}\end{array}$ & $\begin{array}{c}\text { Crushing strength } \\
(\mathrm{N}) \pm \mathrm{SD}\end{array}$ & $\begin{array}{c}\text { Friability (\%) } \\
\pm \mathrm{SD}\end{array}$ & $\begin{array}{c}\text { Amlodipine } \\
\text { recovery }(\%)\end{array}$ & $\begin{array}{c}\text { Lisinopril } \\
\text { recovery (\%) }\end{array}$ \\
\hline A2L4 & $170.5 \pm 1.08$ & $>483.7 \pm 0.58$ & $0.23 \pm 0.05$ & $103.9 \pm 4.7$ & $96.9 \pm 0.7$ \\
A3L6 & $168.6 \pm 5.71$ & $>484.0 \pm 0.00$ & $0.57 \pm 0.48$ & $99.1 \pm 7.4$ & $99.9 \pm 0.3$ \\
A4L8 & $163.6 \pm 3.65$ & $>483.7 \pm 0.58$ & $0.93 \pm 0.90$ & $103.1 \pm 2.4$ & $98.7 \pm 7.6$ \\
\hline
\end{tabular}


336 In order to facilitate the integration of 3D printing for the production of 337 antihypertensive polyprintlets at the point-of-care, a non-destructive method is 338 required to enable at-line quality control and batch release. Previously, we have 339 proven the feasibility of using process analytical technologies (PAT) to quantify 340 a single model drug (paracetamol) in SLS printlets (Trenfield et al., 2018b). 341 Here, we have investigated the use of a portable NIR spectrometer to quantify 342 both therapeutically-relevant dosages of amlodipine and lisinopril in 3D printed 343 oral films and cylindrical tablets. Initially, the pure drugs (amlodipine and 344 lisinopril) and pure PEO 100,000 were scanned to identify unique peaks of 345 interest for calibration model development (Figure 4). For amlodipine, the 346 wavelengths selected ranged between 1450-1600 nm and 2000-2100 nm, 347 whereas for lisinopril the wavelength selected was between 1600-1730 nm. 348 Lisinopril also displayed a high absorbance at $\sim 1920 \mathrm{~nm}$, however this peak is 349 attributed to the presence of water due to the drug being in the dihydrate form; as such, this peak was excluded for model development. The feasibility of using the selected absorbance peaks was evaluated by scanning formulation blends of increasing drug concentrations (amlodipine: 1-4\% $w / w$ and lisinopril 2-8\% $w / w)$ (Figure 5). The NIR absorbance was found to increase upon increasing concentrations of both amlodipine (Figures 5A and B) and lisinopril (Figure 5C) formulations, indicating their suitability for calibration model development.

Figure 4. Second derivative spectra Wavelength (nm)

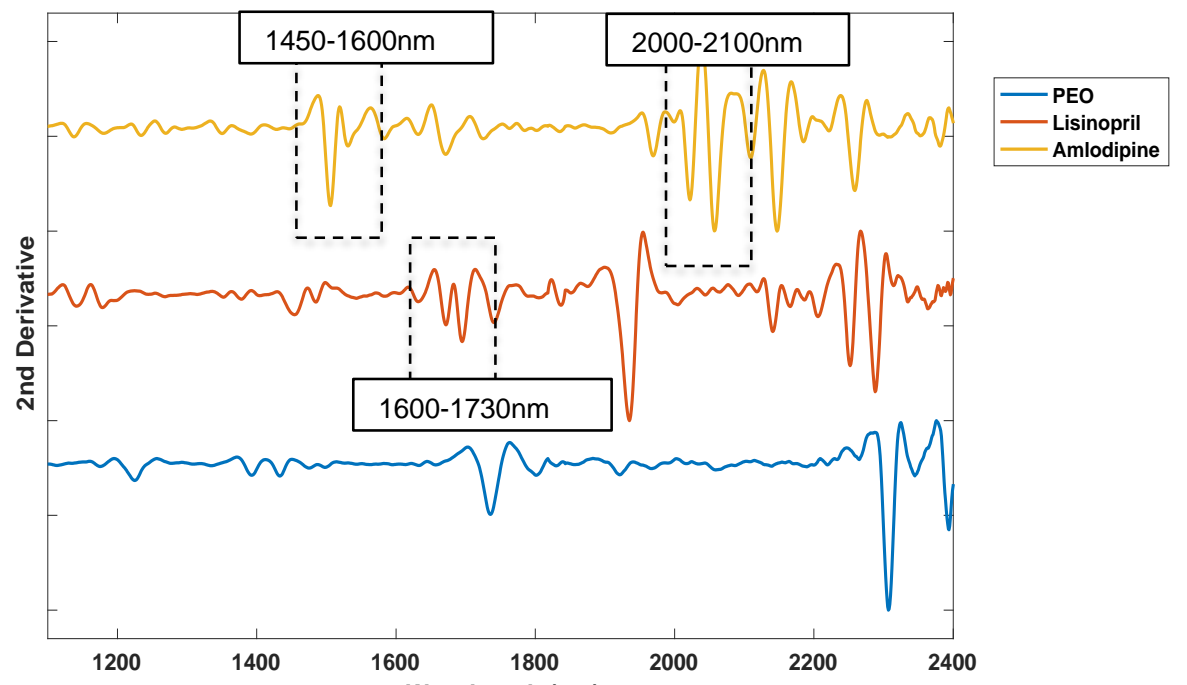
amlodipine. Spectral features of interest were 1450-1600 nm and 2000-2100 nm for amlodipine, and 1600-1730 $\mathrm{nm}$ for lisinopril. 
370 Data pre-treatment is essential to eliminate or minimise variability unrelated to 371 the property of interest and to minimise physical effects prior to multivariate 372 calibration, to ensure the development of an effective model (Huang et al., 373 2010). Pre-treatment improves the accuracy of quantification by enhancing 374 spectral information and reducing baseline drift (Chalus et al., 2005). Evaluation 375 of a variety of pre-processing methods was performed to create a reliable 376 multivariate calibration model (data not shown). In this study, for amlodipine, 377 the model selected has 4 latent variables (LVs), covers between 1450-1600 nm 378 and 2000-2100 $\mathrm{nm}$ wavelength range with a second derivative (Savitzky and 379 Golay method: filter width of 21 with a second polynomial (Savitzky and Golay, 380 1964)), followed by multiplicative scatter correction (MSC) and mean centering 381 pre-processing techniques. 

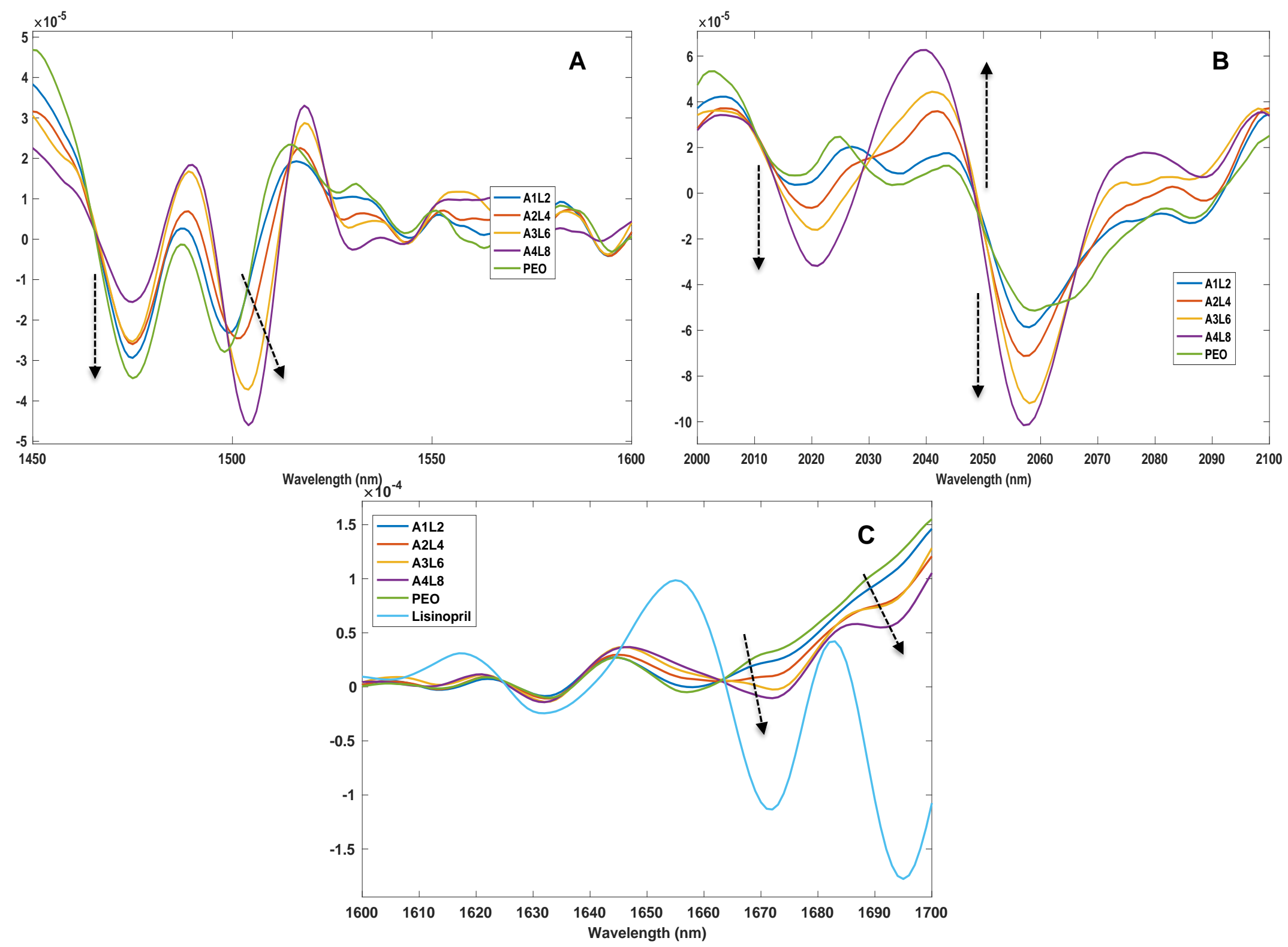
404 Figure 5. Change in second derivative NIR absorbances with the concentration of amlodipine in A) 1450-1600 nm, B) $2000-2100 \mathrm{~nm}$ and lisinopril in C) 1600 $4051700 \mathrm{~nm}$ 
406 For lisinopril, the model selected had 4 LVs, and covers a wavelength range

407 between 1600-1730 nm with a second derivative (Savitzky and Golay method:

408 filter width of 15 with a second polynomial (Savitzky and Golay, 1964)), followed

409 by standard normal variant (SNV) and mean centering pre-processing

410 techniques. These models were selected due to having a high linearity $\left(\mathrm{R}^{2}=\right.$

4110.997 for amlodipine; 0.991 for lisinopril) and high accuracy (RMSEP $=0.24 \%$

412 for amlodipine; $0.24 \%$ for lisinopril) (Figures $6 \mathrm{~A}$ and $\mathrm{B}$ ). These values confirmed

413 that the NIR test results were proportional to the amlodipine and lisinopril

414 concentrations in the stated range. There are several parameters including

415 model linearity, specificity and accuracy that are recommended by $\mathrm{ICH}(\mathrm{ICH}$,

416 1994a), EMA (EMA, 2014a) and FDA (FDA, 2015b) guidelines that the

417 developed models need to satisfy to be validated.

418
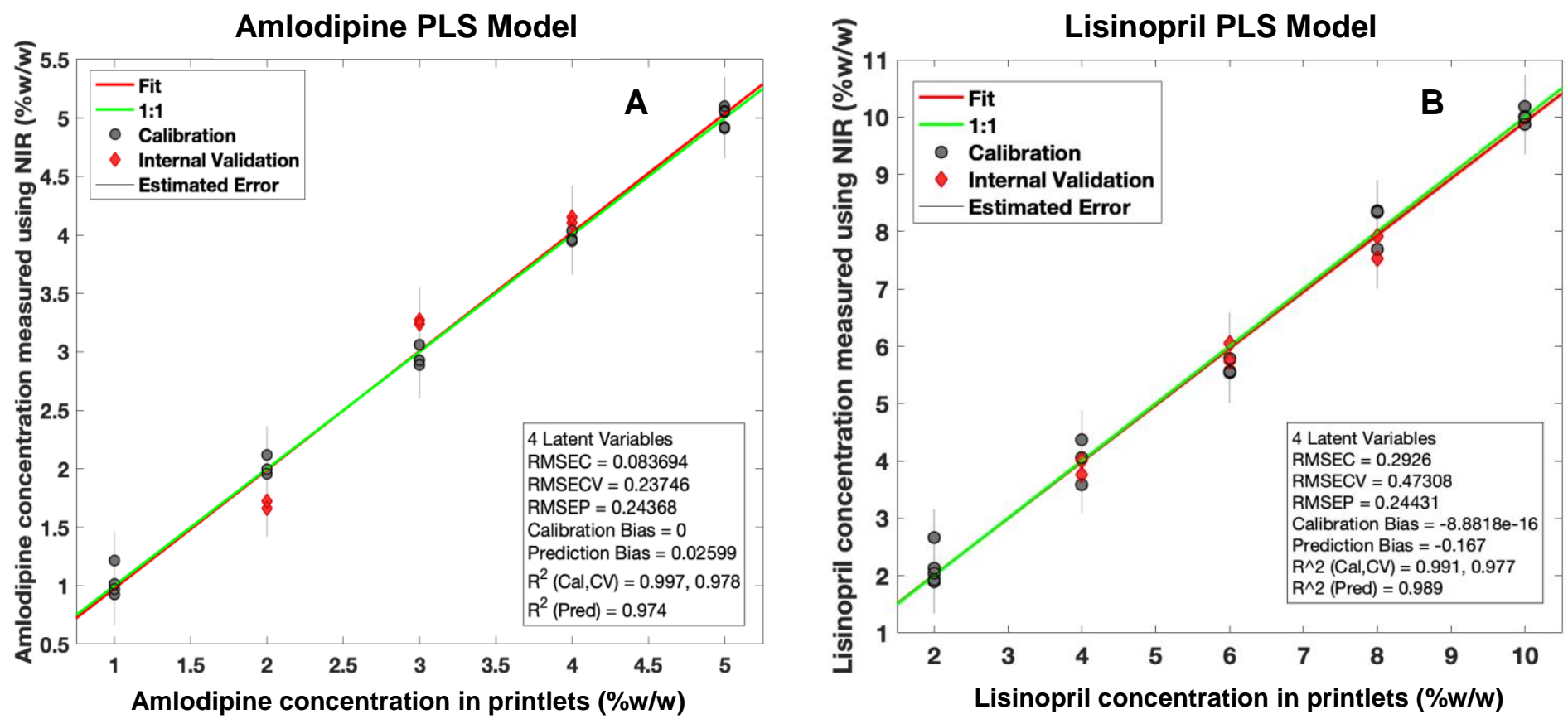

431 Figure 6. PLS calibration models of NIR predicted drug content $(\% \mathrm{w} / \mathrm{w})$ of oral film printlets for

432 A) Amlodipine and B) Lisinopril. Grey points are calibration (19 points from 5 concentrations);

433 Red points are internal validation (6 points from 3 concentrations).

434

435

436

437

438 


\subsubsection{Specificity}

441 Specificity is defined as the ability to identify definitely the analyte (amlodipine 442 and lisinopril) from the other excipients (Candurin Gold Sheen and PEO 443 100,000) (Patel et al., 2012). This was evaluated by comparing the loadings 444 spectra of the $1^{\text {st }}$ latent variable (LV 1) to the pure material reference spectra, 445 which accounted for variation of $82.77 \%$ and $79.55 \%$ for amlodipine and 446 lisinopril, respectively (Figure 7). The LV1 spectrum was found to model well447 known amlodipine spectral features at 1450-1600 nm and 2000-2100 nm 448 (demonstrated in Figures 7A and C) and well-known lisinopril spectral features 449 at 1600-1730 nm (highlighted in Figures 7B and D). The LV1 spectra of both 450 amlodipine and lisinopril models were not found to be modelling common 451 spectral features of PEO 100,0 00 (Figure 4).
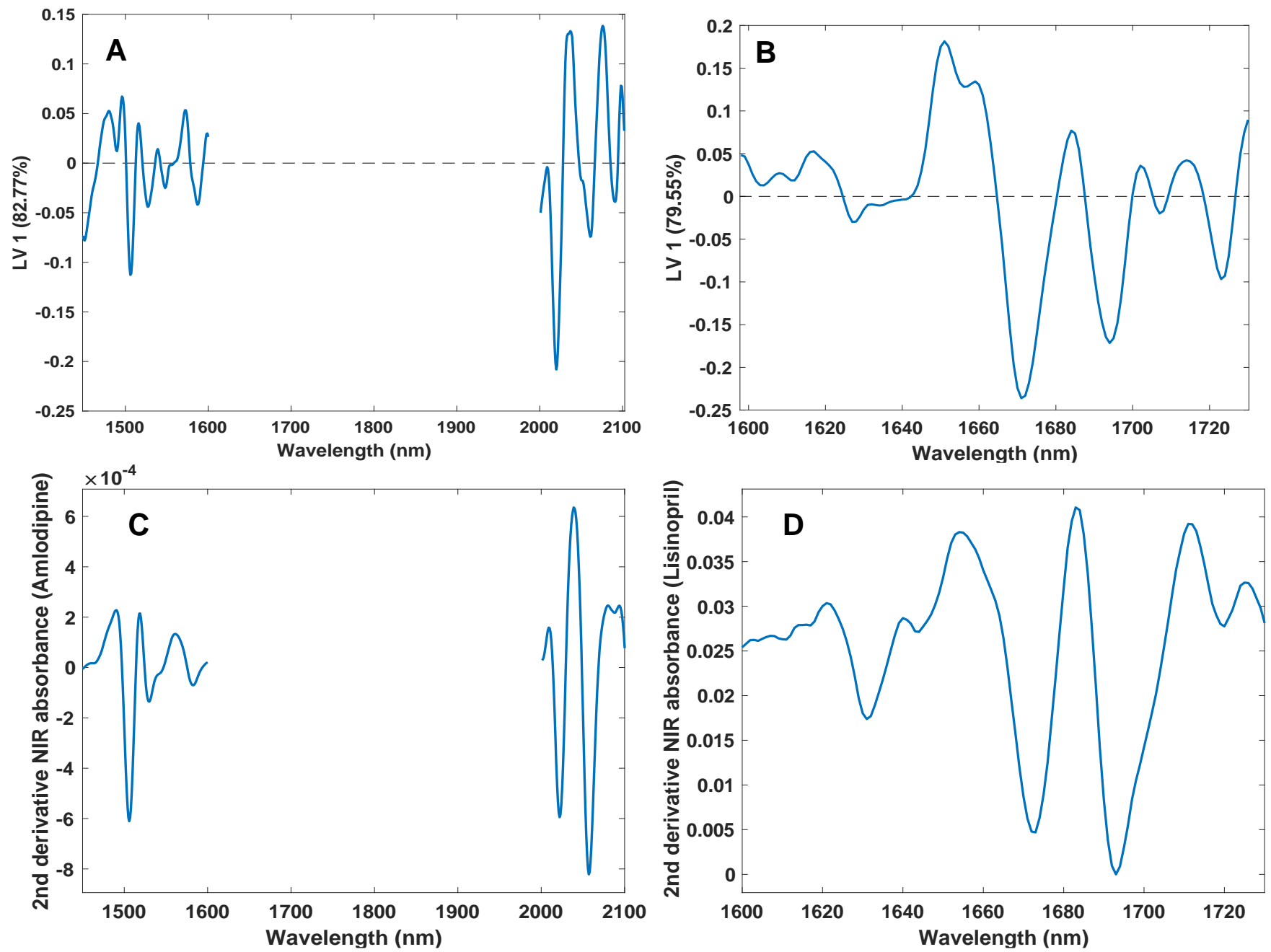

453 Figure 7. NIR absorbance spectra of A) Amlodipine PLS model LV1 loading spectra, B) 454 Lisinopril PLS model LV1 loading spectra, C) Amlodipine pure and D) Lisinopril pure 


\subsubsection{Accuracy}

456 The accuracy of a calibration model can be defined as the closeness in the agreement between the actual and the predicted NIR values $(\mathrm{ICH}, 1994 \mathrm{a})$. As such, oral film printlets from 3 concentration levels (A2L4, A3L6 and A4L8) were scanned and the model was evaluated for prediction accuracy. An excellent predictive performance was observed with both drugs as the RMSEP for amlodipine was $0.24 \%$ and RMSEP was $0.24 \%$ for lisinopril (Figures $6 \mathrm{~A}$ and $\mathrm{B}$ ). Table 3 shows the difference between the HPLC and NIR predicted amlodipine and lisinopril concentrations. Paired t-test results showed that there were no significant differences between HPLC and NIR predictions as $p>0.05$ across all three concentrations. This confirmed that NIR is a suitable quantification method for standard printlets. NIR prediction showed a higher SD compared to HPLC, which may be due to the minute differences in the surface effects of the printlets (Trenfield et al., 2018b). Generally, the model maintained a good predictive performance due to a majority of the data variation being attributed to the changes in the drugs' concentration (LV1 for amlodipine $=82.77 \%$; LV1 for lisinopril $=79.55 \%$ ).

Table 3. Results of dose predicted from oral film printlets test set using the NIR model vs the reference HPLC method

\begin{tabular}{cccc|ccc}
\hline Formulation & \multicolumn{6}{c}{ Test Validation } \\
\cline { 2 - 7 } & \multicolumn{2}{c}{ Amlodipine (\% w/w) } & \multicolumn{3}{c}{ Lisinopril $(\%$ w/w) } \\
\cline { 2 - 7 } & HPLC & NIR & P & HPLC & NIR & P \\
& & & value & & & value \\
\hline A2L4 & $2.08 \pm 0.001$ & $1.99 \pm 0.09$ & 0.23 & $2.90 \pm 0.0001$ & $3.03 \pm 0.27$ & 0.54 \\
A3L6 & $2.97 \pm 0.002$ & $2.62 \pm 0.12$ & 0.10 & $5.99 \pm 0.0001$ & $5.72 \pm 0.25$ & 0.26 \\
A4L8 & $4.13 \pm 0.000$ & $3.73 \pm 0.31$ & 0.15 & $7.90 \pm 0.006$ & $7.46 \pm 0.46$ & 0.41 \\
\hline
\end{tabular}

477 One of the main benefits of 3D printing for personalised medicine is the ability 478 to tailor solid dosage form shape and size, depending on the patient preference 479 or therapeutic needs (Trenfield et al., 2018a). Several studies have already 480 shown that changing printlet geometry can alter the dose and drug release 481 characteristics (Goyanes et al., 2015b; Martinez et al., 2018; Sadia et al., 
2018a). It is also well known that NIR absorbance can be affected by surface

483 effects (Jamrógiewicz, 2012; Saeed et al., 2009) and, as such, it was important

484 to evaluate the performance of the developed PLS model when scanning 485 printlets of different geometries.

486 To determine this, cylindrical shaped printlets (amlodipine 2-4\% $w / w$ and 487 lisinopril $4-8 \% w / w)$ with the same formulation compositions as the oral films were 3D printed and scanned using NIR at six different points on the dosage form. Figures $8 \mathrm{~A}$ and $8 \mathrm{~B}$ show that the model predicted the concentrations well, 490 as the points fitted on the calibration curve. For amlodipine prediction, the 491 change in printlet geometry from a thin film to cylindrical tablet caused a slightly 492 higher error compared to oral films (RMSEP values of $0.26 \%$ and $0.24 \%$, 493 respectively). A similar occurrence observed with the prediction of lisinopril, 494 with RMSEP values of $0.77 \%$ and $0.24 \%$ for cylindrical tablets and oral films, 495 respectively. This phenomenon is likely due to the complex rounded surface 496 structure of this shape compared with the flat films. However, overall the model 497 continued to be fit-for-purpose for use with differently shaped tablets of the 498 same composition.

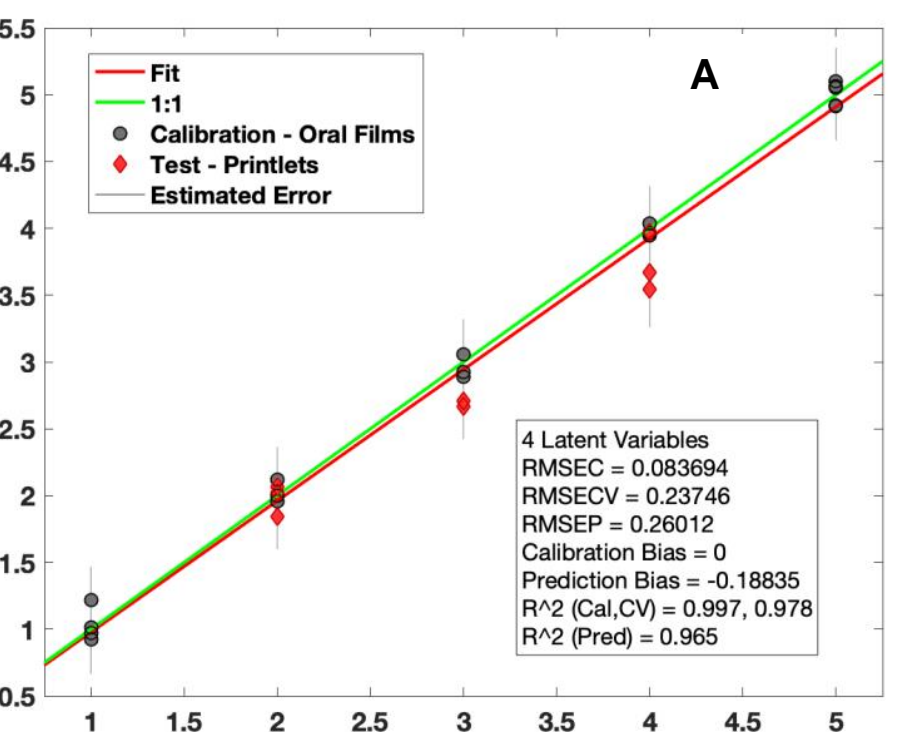

50

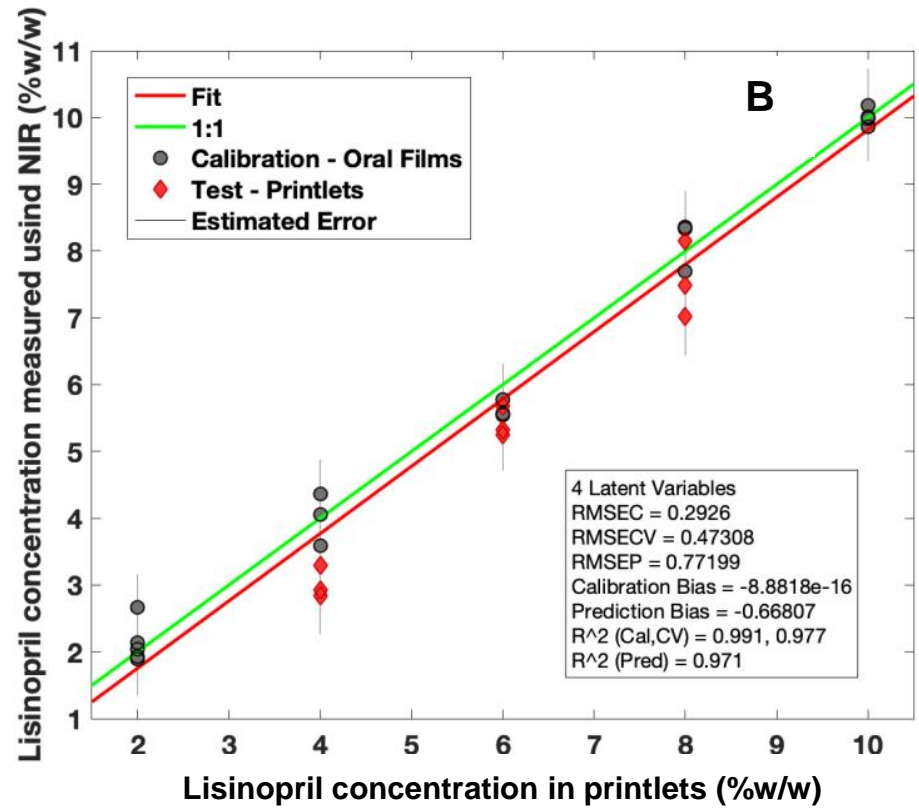

Lisinopril concentration in printlets $(\% \mathrm{w} / \mathrm{w})$

509 Figure 8. Application of developed PLS models of NIR predicted drug content of A) amlodipine

510 and B) lisinopril. Grey points are calibration (based on oral films); red points are a test set of 511 cylindrical printlets. 
512 Currently, PLS regression is widely used for a full quantitative characterisation

513 as it gives the highly accurate predictions (Ravn et al., 2008; Roggo et al., 2007).

514 The non-destructive, at-line QC method demonstrated here clearly shows a lot

515 of advantages as it is highly user-friendly and provides rapid dose prediction

516 with the scanning time for each tablet is only roughly 10 seconds. Since the

517 FDA and EMA guidance also recognise the use of PLS regression as a

518 quantitative tool, the developed model in the present study is suitable to be

519 used for quality control purposes in the clinic (EMA, 2014a; FDA, 2015b). The

520 validation of the developed PLS models have proven the feasibility of the use

521 of NIR spectroscopy to replace conventional destructive dose verification

522 methods (such as HPLC and UV spectroscopy).

\section{Conclusion}

526 For the first time, we report the at-line dose verification of two separate drugs

527 (amlodipine and lisinopril) within 3D printed antihypertensive polyprintlets.

528 Calibration models were developed across therapeutically relevant dosages of 529 two drugs (amlodipine: $1-5 \% \mathrm{w} / \mathrm{w}$, and lisinopril 2-10\% w/w) and were 530 applicable to polyprintlets of different geometries (oral films and cylindrical 531 tablets). The developed models demonstrated excellent linearity $\left(R^{2}\right.$ pred $=$ $5320.997,0.991)$, accuracy (RMSEP $=0.24 \%, 0.24 \%$ ) and specificity (LV1 =

$53382.77 \%, 79.55 \%)$ for both amlodipine and lisinopril respectively, and were 534 validated according to current international standards. This manuscript 535 provides a novel method for the dual quantification of two drugs, facilitating the 536 integration of 3D printing into clinical practice.

\section{Acknowledgements}

540 The authors thank the Engineering and Physical Sciences Research Council

541 (EPSRC), UK for their financial support (EP/L01646X). 
547 Abegaz, T.M., Shehab, A., Gebreyohannes, E.A., Bhagavathula, A.S., Elnour,

548 A.A., 2017. Nonadherence to antihypertensive drugs: A systematic review and 549 meta-analysis. Medicine 96, e5641.

550 Alhnan, M.A., Okwuosa, T.C., Sadia, M., Wan, K.W., Ahmed, W., Arafat, B.,

551 2016. Emergence of 3D Printed Dosage Forms: Opportunities and

552 Challenges. Pharm Res 33, 1817-1832.

553 Alomari, M., Vuddanda, P.R., Trenfield, S.J., Dodoo, C.C., Velaga, S., Basit,

554 A.W., Gaisford, S., 2018. Printing T3 and T4 oral drug combinations as a

555 novel strategy for hypothyroidism. International Journal of Pharmaceutics 549, 556 363-369.

557 Awad, A., Fina, F., Trenfield, S.J., Patel, P., Goyanes, A., Gaisford, S., Basit,

558 A.W., 2019. 3D Printed Pellets (Miniprintlets): A Novel, Multi-Drug, Controlled

559 Release Platform Technology. Pharmaceutics 11(4), 148.

560 Awad, A., Trenfield, S.J., Gaisford, S., Basit, A.W., 2018a. 3D printed

561 medicines: A new branch of digital healthcare. Int J Pharm 548, 586-596.

562 Awad, A., Trenfield, S.J., Goyanes, A., Gaisford, S., Basit, A.W., 2018b.

563 Reshaping drug development using 3D printing. Drug Discovery Today 23,

564 1547-1555.

565 Babu, N.J., Nangia, A., 2011. Solubility Advantage of Amorphous Drugs and 566 Pharmaceutical Cocrystals.

567 Barakh Ali, S.F., Mohamed, E.M., Ozkan, T., Kuttolamadom, M.A., Khan,

568 M.A., Asadi, A., Rahman, Z., 2019. Understanding the effects of formulation

569 and process variables on the printlets quality manufactured by selective laser

570 sintering 3D printing. Int J Pharm 570, 118651.

571 Barnatt, C., 2013. 3D Printing: The Next Industrial Revolution.

572 Basit, A., Gaisford, S., 2018. 3D Printing of Pharmaceuticals. DOI:

573 https://doi.org/10.1007/978-3-319-90755-0. Springer.

574 BP, 2018. Appendix XVII G. Friability - British Pharmacopoeia.

575 Capretto, L., Byrne, G.T., S, L, D., 2017. Formulation, Analytical, and

576 Regulatory Strategies for First-in-Human Clinical Trials, in: E, K. (Ed.), Oral

577 Formulation Roadmap from Early Drug Discovery to Development. 
578 Chalus, P., Roggo, Y., Walte, r.S., Ulmschneider, M., 2005. Near-infrared

579 determination of active substance content in intact low-dosage tablets. -

580 Abstract - Europe PMC.

581 Corrêa, N.B., de Faria, A.P., Ritter, A.M.V., Sabbatini, A.R., Almeida, A.,

582 Brunelli, V., Calhoun, D.A., Moreno, H., Modolo, R., 2016. A practical

583 approach for measurement of antihypertensive medication adherence in

584 patients with resistant hypertension. Journal of the American Society of

585 Hypertension 10, 510-516.e511.

586 Di Prima, M., Coburn, J., Hwang, D., Kelly, J., Khairuzzaman, A., Ricles, L.,

587 2016. Additively manufactured medical products - the FDA perspective |

588 SpringerLink.

589 DrugBank, Lisinopril dihydrate.

590 Durden, M., Avery, T., Rupert, P., 2013. Polypharmacy and medicines

591 optimisation. @TheKingsFund.

592 Edinger, M., Iftimi, L.-D., Markl, D., Al-Sharabi, M., Bar-Shalom, D., Rantanen,

593 J., Genina, N., 2019. Quantification of Inkjet-Printed Pharmaceuticals on

594 Porous Substrates Using Raman Spectroscopy and Near-Infrared

595 Spectroscopy. AAPS PharmSciTech 20, 207.

596 Edinger, M., Jacobsen, J., Bar-Shalom, D., Rantanen, J., Genina, N., 2018.

597 Analytical aspects of printed oral dosage forms. International Journal of

598 Pharmaceutics 553, 97-108.

599 EMA, 2012. Guideline on Real Time Release Testing (formerly Guideline on

600 Parametric Release).

601 EMA, 2014a. Use of near infrared spectroscopy (NIRS) by the pharmaceutical

602 industry and the data requirements for new submissions and variations |

603 European Medicines Agency.

604 EMA, E.M.A., 2014b. Use of near infrared spectroscopy (NIRS) by the

605 pharmaceutical industry and the data requirements for new submissions and

606 variations. European Medicines Agency.

607 FDA, 2015a. Development and Submission of Near Infrared Analytical

608 Procedures; Draft Guidance for Industry; Availability.

609 FDA, 2015b. Development and Submission of Near Infrared Analytical

610 Procedures; Draft Guidance for Industry; Availability. 
611 Fina, F., Goyanes, A., Gaisford, S., Basit, A.W., 2017. Selective laser

612 sintering (SLS) 3D printing of medicines. Int J Pharm 529, 285-293.

613 Fina, F., Goyanes, A., Madla, C.M., Awad, A., Trenfield, S.J., Kuek, J.M.,

614 Patel, P., Gaisford, S., Basit, A.W., 2018a. 3D printing of drug-loaded gyroid

615 lattices using selective laser sintering. International journal of pharmaceutics $616547,44-52$.

617 Fina, F., Madla, C.M., Goyanes, A., Zhang, J., Gaisford, S., Basit, A.W.,

618 2018b. Fabricating 3D printed orally disintegrating printlets using selective 619 laser sintering. Int J Pharm 541, 101-107.

620 Florence, A.T., Lee, V.H., 2011. Personalised medicines: more tailored drugs,

621 more tailored delivery. Int J Pharm 415, 29-33.

622 Genina, N., Boetker, J.P., Colombo, S., Harmankaya, N., Rantanen, J., Bohr, 623 A., 2017. Anti-tuberculosis drug combination for controlled oral delivery using $6243 \mathrm{D}$ printed compartmental dosage forms: From drug product design to in vivo 625 testing. Journal of Controlled Release 268, 40-48.

626 Gioumouxouzis, C.I., Baklavaridis, A., Katsamenis, O.L., Markopoulou, C.K., 627 Bouropoulos, N., Tzetzis, D., Fatouros, D.G., 2018. A 3D printed bilayer oral 628 solid dosage form combining metformin for prolonged and glimepiride for 629 immediate drug delivery. Eur J Pharm Sci 120, 40-52.

630 Goyanes, A., Allahham, N., Trenfield, S.J., Stoyanov, E., Gaisford, S., Basit, 631 A.W., 2019a. Direct powder extrusion 3D printing: Fabrication of drug 632 products using a novel single-step process. International Journal of 633 Pharmaceutics 567, 118471.

634 Goyanes, A., Buanz, A.B.M., Hatton, G.B., Gaisford, S., Basit, A.W., 2015a. $6353 \mathrm{D}$ printing of modified-release aminosalicylate (4-ASA and 5-ASA) tablets. 636 European Journal of Pharmaceutics and Biopharmaceutics 89, 157-162.

637 Goyanes, A., Madla, C.M., Umerji, A., Duran Pineiro, G., Giraldez Montero, 638 J.M., Lamas Diaz, M.J., Gonzalez Barcia, M., Taherali, F., Sanchez-Pintos, 639 P., Couce, M.L., Gaisford, S., Basit, A.W., 2019b. Automated therapy 640 preparation of isoleucine formulations using 3D printing for the treatment of 641 MSUD: First single-centre, prospective, crossover study in patients. Int J 642 Pharm 567, 118497. 
643 Goyanes, A., Robles Martinez, P., Buanz, A., Basit, A.W., Gaisford, S.,

$6442015 b$. Effect of geometry on drug release from 3D printed tablets.

645 International Journal of Pharmaceutics 494, 657-663.

646 Goyanes, A., Scarpa, M., Kamlow, M.-A., Gaisford, S., Basit, A., Orlu, M., 647 2017. Patient acceptability of 3D printed medicines. International Journal of 648 Pharmaceutics 530.

649 Hamburg, M.A., Collins, F.S., 2010. The path to personalized medicine. N 650 Engl J Med 363, 301-304.

651 Herttua, K., Tabak, A.G., Martikainen, P., Vahtera, J., Kivimaki, M., 2013.

652 Adherence to antihypertensive therapy prior to the first presentation of stroke

653 in hypertensive adults: population-based study. Eur Heart J 34, 2933-2939.

654 Hinojosa-Torres, J., Aceves-Hernández, J.M., Hinojosa-Torres, J., Paz, M., 655 Castaño, V.M., 2008. Degradation of lisinopril: A physico-chemical study. 656 Journal of Molecular Structure 886, 51-58.

657 Huang, J., Romero-Torres, S., Moshgbar, M., 2010. Practical Considerations 658 in Data Pre-treatment for NIR and Raman Spectroscopy.

$659 \mathrm{ICH}, 1994 \mathrm{a}$. Validation of Analytical Procedures: Text and Methodology.

$660 \mathrm{ICH}, 1994 \mathrm{~b}$. Validation of Analytical Procedures: Text and Methodology, in:

661 Group, I.E.W. (Ed.), International Conference On Harmonisation Of Technical

662 Requirements For Registration Of Pharmaceuticals For Human Use, pp. 1-17.

663 Jamrógiewicz, M., 2012. Application of the near-infrared spectroscopy in the

664 pharmaceutical technology. Journal of pharmaceutical and biomedical 665 analysis $66,1-10$.

666 Khaled, S.A., Burley, J.C., Alexander, M.R., Yang, J., Roberts, C.J., 2015a.

667 3D printing of five-in-one dose combination polypill with defined immediate 668 and sustained release profiles. J Control Release 217, 308-314.

669 Khaled, S.A., Burley, J.C., Alexander, M.R., Yang, J., Roberts, C.J., 2015b.

$6703 \mathrm{D}$ printing of tablets containing multiple drugs with defined release profiles.

671 Int J Pharm 494, 643-650.

672 Kollamaram, G., Croker, D.M., Walker, G.M., Goyanes, A., Basit, A.W.,

673 Gaisford, S., 2018. Low temperature fused deposition modeling (FDM) 3D

674 printing of thermolabile drugs. undefined. 
675 Lee, H.J., Jang, S.-I., Park, E.-C., 2017. Effect of adherence to

676 antihypertensive medication on stroke incidence in patients with hypertension:

677 a population-based retrospective cohort study. BMJ Open 7, e014486.

678 Martinez, L.M., Videa, M., Lopez-Silva, G.A., de Los Reyes, C.A., Cruz-

679 Angeles, J., Gonzalez, N., 2014. Stabilization of amorphous paracetamol

680 based systems using traditional and novel strategies. Int J Pharm 477, 294-

681305.

682 Martinez, P.R., Goyanes, A., Basit, A.W., Gaisford, S., 2018. Influence of

683 Geometry on the Drug Release Profiles of Stereolithographic (SLA) 3D-

684 Printed Tablets. AAPS PharmSciTech. https://doi.org/10.1208/s12249-018-

$685 \quad$ 1075-3.

686 NICE, 2011. Hypertension in adults: diagnosis and management.

687 Oblom, H., Zhang, J., Pimparade, M., Speer, I., Preis, M., Repka, M., Sandler,

688 N., 2019. 3D-Printed Isoniazid Tablets for the Treatment and Prevention of

689 Tuberculosis-Personalized Dosing and Drug Release. AAPS PharmSciTech

$69020,52$.

691 Ong, K.L., Cheung, B.M., Man, Y.B., Lau, C.P., Lam, K.S., 2007. Prevalence,

692 awareness, treatment, and control of hypertension among United States

693 adults 1999-2004. Hypertension 49, 69-75.

694 Patel, D.B., Mehta, F.A., Bhatt, K.K., 2012. Simultaneous Estimation of

695 Amlodipine Besylate and Indapamide in a Pharmaceutical Formulation by a

696 High Performance Liquid Chromatographic (RP-HPLC) Method. Sci Pharm

$69780,581-590$.

698 Pereira, B., Isreb, A., Forbes, R., Dores, F., Habashy, R., Petit, J.-B., A

699 Alhnan, M., Oga, E., 2018. 'Temporary Plasticiser': A Novel Solution to

700 Fabricate 3D Printed Patient-Centred Cardiovascular 'Polypill' Architectures.

701 European Journal of Pharmaceutics and Biopharmaceutics 135.

702 Pubchem, 2003. Amlodipine.

703 Pubchem, 2004. Ethylene glycol.

704 Pubchem, 2005. Lisinopril.

705 Ravn, C., Skibsted, E., Bro, R., 2008. Near-infrared chemical imaging (NIR-

$706 \mathrm{Cl}$ ) on pharmaceutical solid dosage forms-comparing common calibration

707 approaches. J Pharm Biomed Anal 48, 554-561. 
708 Robles-Martinez, P., Xu, X., Trenfield, S.J., Awad, A., Goyanes, A., Telford, 709 R., Basit, A.W., Gaisford, S., 2019. 3D Printing of a Multi-Layered Polypill

710 Containing Six Drugs Using a Novel Stereolithographic Method.

711 Pharmaceutics 11, 274.

712 Roggo, Y., Chalus, P., Maurer, L., Lema-Martinez, C., Edmond, A., Jent, N., 713 2007. A review of near infrared spectroscopy and chemometrics in 714 pharmaceutical technologies. J Pharm Biomed Anal 44, 683-700.

715 Roy, A., Naik, N., Srinath Reddy, K., 2017. Strengths and Limitations of Using 716 the Polypill in Cardiovascular Prevention. Current Cardiology Reports 19, 45.

717 Ruilope, L.M., 2011. Current challenges in the clinical management of 718 hypertension. Nat Rev Cardiol 9, 267-275.

719 Sadia, M., Arafat, B., Ahmed, W., Forbes, R.T., Alhnan, M.A., 2018a.

720 Channelled tablets: An innovative approach to accelerating drug release from 721 3D printed tablets. J Control Release 269, 355-363.

722 Sadia, M., Isreb, A., Abbadi, I., Isreb, M., Aziz, D., Selo, A., Timmins, P.,

723 Mohammed, A., 2018b. From 'fixed dose combinations' to 'a dynamic dose

724 combiner': 3D printed bi-layer antihypertensive tablets. 484-494.

725 Sadia, M., Sosnicka, A., Arafat, B., Isreb, A., Ahmed, W., Kelarakis, A., 726 Alhnan, M.A., 2016. Adaptation of pharmaceutical excipients to FDM 3D 727 printing for the fabrication of patient-tailored immediate release tablets. Int $\mathrm{J}$ 728 Pharm 513, 659-668.

729 Saeed, M., Saner, S., Oelichmann, J., Keller, H., Betz, G., 2009. Assessment 730 of Diffuse Transmission Mode in Near-Infrared Quantification-Part I: The 731 Press Effect on Low-Dose Pharmaceutical Tablets. Journal of Pharmaceutical 732 Sciences 98, 4877-4886.

733 Savitzky, A., Golay, M.J.E., 1964. Smoothing and Differentiation of Data by 734 Simplified Least Squares Procedures. Analytical Chemistry 36, 1627-1639.

735 Tibebu, A., Mengistu, D., Bulto, L.N., 2017. Adherence to prescribed 736 antihypertensive medications and associated factors for hypertensive patients 737 attending chronic follow-up units of selected public hospitals in Addis Ababa, 738 Ethiopia, Int J Health Sci (Qassim), pp. 47-52.

739 Trenfield, S.J., Awad, A., Goyanes, A., Gaisford, S., Basit, A.W., 2018a. 3D 740 Printing Pharmaceuticals: Drug Development to Frontline Care. Trends in 741 pharmacological sciences 39, 440-451. 
742 Trenfield, S.J., Awad, A., Madla, C.M., Hatton, G.B., Firth, J., Goyanes, A., 743 Gaisford, S., Basit, A.W., 2019a. Shaping the future: recent advances of 3D 744 printing in drug delivery and healthcare. Expert Opinion on Drug Delivery 16, 745 1081-1094.

746 Trenfield, S.J., Goyanes, A., Telford, R., Wilsdon, D., Rowland, M., Gaisford, 747 S., Basit, A.W., 2018b. 3D printed drug products: Non-destructive dose

748 verification using a rapid point-and-shoot approach. Int J Pharm 549, 283-292.

749 Trenfield, S.J., Xian Tan, H., Awad, A., Buanz, A., Gaisford, S., Basit, A.W., 750 Goyanes, A., 2019b. Track-and-Trace: Novel Anti-Counterfeit Measures for 751 3D Printed Personalised Drug Products using Smart Material Inks. Int. J.

752 Pharm. 567, 118443.

753 Vakili, H., Wickstrom, H., Desai, D., Preis, M., Sandler, N., 2017. Application 754 of a handheld NIR spectrometer in prediction of drug content in inkjet printed 755 orodispersible formulations containing prednisolone and levothyroxine. Int J 756 Pharm 524, 414-423.

757 WHO, 2019. Raised Blood Pressure: Global Health Observatory data.

758 Xu, X., Robles-Martinez, P., Madla, C.M., Goyanes, A., Basit, A.W., Gaisford, 759 S., 2020. Stereolithography (SLA) 3D printing of an antihypertensive 760 polyprintlet: Case study of an unexpected photopolymer-drug reaction.

761 Additive Manufacturing DOI: 10.1016/j.addma.2020.101071. 